\title{
Correction to: Efficient multiobjective optimization employing Gaussian processes, spectral sampling and a genetic algorithm
}

\author{
Eric Bradford $^{1,3} 3_{(\mathbb{D})} \cdot$ Artur M. Schweidtmann $^{2,3}$ ・ \\ Alexei Lapkin ${ }^{3}$
}

Published online: 9 March 2018

(C) Springer Science+Business Media, LLC, part of Springer Nature 2018

\section{Correction to: J Glob Optim https://doi.org/10.1007/s10898-018-0609-2}

This note lists changes to the article by Bradford et al. [1]. A number of figures have moved to the wrong positions, while the references in the text still refer to the correct figure, i.e. a number of figures are now in the wrong section, have the wrong position and have the wrong caption.

- The following corrections are required for the cross-references in the text:

- Delete "The results of the hypervolume indicator are shown in Fig. 5." at the bottom of Sect. 8.2.1.

- Delete "The results of the modified inverted generational distance indicator are shown in Fig. 6." at the bottom of Sect. 8.2.2.

- Delete "The results of the generalized spread indicator are shown in Fig. 7." at the bottom of Sect. 8.2.3

- Change "In Fig. 8 on the right-hand side we have schematically plotted 5 attainment surfaces." in Sect. 8.2.4 to "In Fig. 5 on the right-hand side we have schematically plotted 5 attainment surfaces." at the same position.

The original article can be found online at https://doi.org/10.1007/s10898-018-0609-2.

Eric Bradford

ecb65@cantab.net

Alexei Lapkin

aal35@cam.ac.uk

1 Department of Engineering Cybernetics, Norwegian University of Science and Technology, Trondheim 7491, Norway

2 Aachener Verfahrenstechnik - Process Systems Engineering, RWTH Aachen University, Aachen 52062, Germany

3 Department of Chemical Engineering and Biotechnology, University of Cambridge, Cambridge CB2 3RA, UK 
- New paragraph after "In Fig. 8 on the right-hand side we have schematically plotted 5 attainment surfaces." in Sect. 8.2.4 with references to figures:

- The results of the hypervolume indicator are shown in Fig. 6, the results of the modified inverted generational distance indicator are shown in Fig. 7 and the results of the generalized spread indicator are shown in Fig. 8.

- The position of the figures are changed as follows:

- Fig. 5 to the position of Fig. 6 (i.e. Figure 5 becomes Fig. 6)

- Fig. 6 to the position of Fig. 7 (i.e. Figure 6 becomes Fig. 7)

- Fig. 7 to the position of Fig. 8 (i.e. Figure 7 becomes Fig. 8)

- Fig. 8 to the position of Fig. 5 (i.e. Figure 8 becomes Fig. 5)

- The correct captions for the Figs. 5 to 8 after making the above changes are:

- Figure 5 caption: "Illustration of an attainment surface on the left-hand side and on the right-hand side a schematic showing how to plot the worst-attainment summary surface from 5 attainment surfaces"

- Figure 6 caption: "Optimization test problem results shown in boxplots using the hypervolume performance indicator obtained after 20 runs with 150 function evaluations"

- Figure 7 caption: "Optimization test problem results shown in boxplots using the modified inverted generational distance performance indicator obtained after 20 runs with 150 function evaluations"

- Figure 8 caption: "Optimization test problem results shown in boxplots using the generalized spread performance indicator obtained after 20 runs with 150 function evaluations"

\section{Reference}

1. Bradford, E., Schweidtmann, A.M., Lapkin, A.: Efficient multiobjective optimization employing Gaussian processes, spectral sampling and a genetic algorithm. J. Glob. Optim. (2018) 